Clinical microbiology

Clinical microbiology

\title{
Anaerobic blood culture positivity at a University Hospital in Hungary: A 5-year comparative retrospective study
}

\author{
Márió Gajdács ${ }^{\mathrm{a}}$, Marianna Ábrók ${ }^{\mathrm{b}}$, Andrea Lázár ${ }^{\mathrm{b}}$, Gabriella Terhes ${ }^{\mathrm{b}}$, Edit Urbán ${ }^{\mathrm{c}, \text { * }}$ \\ a Department of Pharmacodynamics and Biopharmacy, Faculty of Pharmacy, University of Szeged, 6720 Szeged, Eötvös utca 6, Szeged, Hungary \\ ${ }^{\mathrm{b}}$ Institute of Clinical Microbiology, Faculty of Medicine, University of Szeged, 6725 Szeged, Semmelweis utca 6, Szeged, Hungary \\ ${ }^{c}$ Department of Public Health, Faculty of Medicine, University of Szeged, 6720 Szeged, Dóm tér 10, Szeged, Hungary
}

\section{A R T I C L E IN F O}

\section{Article history:}

Received 5 January 2020

Received in revised form 27 March 2020

Accepted 28 March 2020

Available online $\mathrm{xxx}$

Handling Editor: Professor Boyanova

Lyudmila

Keywords:

Bacteremia

Bloodstream infections

Blood cultures

Anaerobes

MALDI-TOF

Bacteroides

Clostridium

\begin{abstract}
A B S T R A C T
Anaerobic bacteremia (AB) is usually detected in about $0.5-13 \%$ of positive blood cultures. The aim of this study was to determine prevalence of anaerobic bacteremia over a 5-year period (2013-2017), to identify current trends at our University Hospital and to compare the results to those in a similar study (2005-2009) in the same region. During the study period, an average of $23,274 \pm 2,756$ blood cultures were received per year. Out of the positive blood cultures, $3.3-3.6 \%(n=423)$ yielded anaerobic bacteria, representing 3.5-3.8 anaerobic isolates/1000 blood culture bottles (including both aerobic and anaerobic bottles) per year for hospitalized patients. Mean age of affected patients was 70-73 years (range: 18-102 years) with a male-to-female ratio: 0.60. Most isolated anaerobes were Cutibacterium spp. $(54.0 \pm 8.5 \% ; \mathrm{n}=247)$, while among anaerobes other than Cutibacterium spp., Bacteroides and Parabacteroides and Clostridium spp. were the most prevalent. Blood culture time-to-positivity (TTP) for clinically relevant bacteria was $31.4 \pm 23.4 \mathrm{~h}$, while for Cutibacterium spp., TTP values were $112.9 \pm 37.2 \mathrm{~h}(\mathrm{p}<0.0001)$. In conclusion, the prevalence of anaerobic bacteremia should be determined on institutional basis.
\end{abstract}

(C) 2020 .

\section{Introduction}

Obligate anaerobic bacteria may be important pathogens at virtually all anatomical sites and are causative agents in multiorgan failure, which can be serious and life-threatening [1]. Because anaerobes are the predominant members of the human microbiome, especially in the intestinal tract and on various mucous membranes, they are a common cause of infections of endogenous origin, in addition to exogenous infections, such as bite wounds and gas gangrene [2]. Anaerobic bacteremia is detected in about $0.5-13 \%$ of all positive blood cultures, mainly depending on the type of the healthcare institution (primary- or tertiary) and/or hospital ward (presence or absence of immunocompromised patients) submitting the blood culture samples $[3,4]$. Due to the variation in clinical and laboratory practices among the different institutions worldwide, potentially inaccurate epidemiological data may be reported. In spite of its infrequent occurrence, the mortality rate associated with anaerobic bacteremia still remains very high (ranging between $25 \%$ and $44 \%$ ) $[1,5,6]$. The characterization of

\footnotetext{
* Corresponding author.

Email address: tidenabru@freemail.hu (E. Urbán)
}

the main risk factors associated with mortality in patients with anaerobic bacteremia was performed in the 1970s. Advanced age, polymicrobial infection and the presence of severe underlying diseases were considered as factors contributing to fatal outcome [7]. The administration of delayed or inappropriate antibiotic therapy against these microorganisms or the lack of surgical intervention may also lead to failures in eradication of these infections [1,7]. The isolation of anaerobic bacteria has specific requirements that should be strictly followed [8]. The management of anaerobic infections is difficult due to the slow growth of many anaerobes, which can delay the identification, the frequent polymicrobial nature of the infections and the increasing resistance of anaerobic bacteria [2]. The number of reports of multidrug-resistant (MDR) anaerobic strains (most often Bacteroides/ Parabacteroides spp.) has increased in the past decade [9]. The significance of inappropriate antimicrobial treatment on the mortality rate of patients with anaerobic bacteremia was highlighted by several studies previously $[10,11]$, although the exact association of these factors was not always conclusive, because inadequate source control (surgical therapy or drainage of abscesses) is also an important point to consider.

There is value in attaining blood both in aerobic and anaerobic blood cultures; clinicians and laboratory personnel at each institution 
should determine the prevalence of anaerobic bacteremia and use this information to guide the practice of taking blood cultures.

The aim of our study was to conduct a 5-year retrospective study to evaluate the incidence of anaerobic bacteremia in hospitalized patients and to establish whether a shift in the frequency or distribution has occurred, compared to a similar study in the same institution, over the same 5-year time period (2005-2009) [12].

\section{Materials and methods}

\subsection{Study design, data collection}

This retrospective observational study was performed on the basis of microbiological data collected, corresponding to a 5-year period (from January 1, 2013 to December 31, 2017). During this time, the Institute of Clinical Microbiology was the National Reference Laboratory of Anaerobic Bacteria in Hungary. The laboratory is the routine diagnostic microbiological laboratory of a (currently) 1,820-bed tertiary-care university-teaching hospital with a broad-profile, affiliated with the University of Szeged in Szeged, Hungary. The Clinical Center is responsible for the medical care of a population of around 600,000 patients in the southeast region of Hungary [13]. The Clinical Center has four adult Intensive Care Units (ICUs) with different profiles: cardiology-hematology, surgery, and traumatology and two ICUs with a pediatric profile (neonatal and pediatric ICU).

Culture results, corresponding to anaerobic blood culture bottles from adult patients ( $\geq 18$ years) were collected by an electronic search of the Institutional laboratory information system (LIS) records for the corresponding 5-year study period. The data collection included data corresponding to samples from inpatient departments (i.e. hospitalized patients) and the emergency department, while samples from outpatient clinics was excluded from the analysis. Isolates were considered separate if they occurred more than 14 days apart [14]. Time-to-positivity (TTP) data corresponding to the positive blood culture bottles was also collected. Polymicrobial bacteremia was defined by the isolation of more than one organism in a single blood culture [14]. In addition, patient data were also collected on patients who had at least one positive blood culture yielding anaerobic bacteria or co-isolation of multiple bacteria involving at least one anaerobic species. The study was deemed exempt from ethics review by the Institutional Review Board and informed consent was not required as patient's data anonymity was maintained.

\subsection{Sample processing and microbial identification}

The processing of blood culture bottles was carried out in accordance with national and international guidelines [15]. With the exception of the department of pediatrics, clinicians routinely used parallel aerobic and anaerobic blood culture bottles in pairs, with most blood cultures ordered as two sets, with one aerobic and one anaerobic bottle per set. Blood cultures were analysed with a BacT/Alert 3D automated system (bioMérieux, Marcy l'Etoile, France) following inoculation of $5-10 \mathrm{~mL}$ of blood into aerobic and anaerobic bottles (BacT/ Alert FA and SN bottles; bioMérieux). Blood culture bottles were incubated with constant shaking for 5 days and for 21 days, if endocarditis was suspected, and monitored in accordance with the manufacturer's instructions.

Samples from positive anaerobic bottles were plated to the Columbia agar base supplemented with $5 \%(\mathrm{v} / \mathrm{v})$ sheep blood (bioMérieux, Marcy l'Etoile, France), and chocolate PolyViteX agar (bioMérieux, Marcy l'Etoile, France) for the cultivation of aerobic bacteria, eosin methylene-blue agar (bioMérieux, Marcy l'Etoile,
France) for the selective growing of Enterobacterales was applied. Samples from the positive bottles were also plated on Schaedler agar (bioMérieux, Marcy l'Etoile, France) containing 5\% v/v horse blood, haemin and Vitamin $\mathrm{K}_{1}$ for the isolation of anaerobic bacteria; these cultures were set up and incubated in an atmosphere of $90 \% \mathrm{~N}_{2}, 5 \%$ $\mathrm{H}_{2}$ and $5 \% \mathrm{CO}_{2}$ in an anaerobic environment (Concept 400 anaerobic incubator, Biotrace International Plc., UK) for $2-5$ days at $37^{\circ} \mathrm{C}$.

Identification of anaerobic isolates was carried out using matrix-assisted laser desorption/ionization time-of-flight mass spectrometry (MALDI-TOF MS), using the microFlex LT Biotyper (Bruker Daltonics Gmbh., Bremen, Germany), the MALDI Biotyper RTC 3.1 software (Bruker Daltonics, Germany) and the MALDI Biotyper Library 3.1 were used for spectrum analysis. Extraction steps with formic acid were carried out before measurements to improve successful identification rates. Methodology of sample preparation and the technical details of the mass spectrometry measurements were described previously [16]. Genus-level identification was considered reliable for $\log$ (score $) \geq 1.7$, while this value for species level identification was $\log$ (score $) \geq 2.0$.

Although Cutibacterium spp. isolates are not considered as causative agents of bacteremia, these isolates were screened, based on the criteria mentioned below to ascertain their possible clinical relevance; isolates were considered clinically-relevant if a) endocarditis was suspected, b) data on previous medical history was suggestive of previous orthopedic surgery or implanted device, c) if Cutibacterium spp. were detected from a pair of blood cultures simultaneously.

\subsection{Statistical and comparative analysis}

Statistical analyses, including descriptive analysis (means or medians with ranges and percentages to characterize data) and statistical tests (Student's t-test and Mann-Whitney $U$ test) were performed with the SPSS software version 24 (IBM SPSS Statistics for Windows 24.0, IBM Corp., Armonk, NY, USA). The normality of variables was tested using Shapiro-Wilk tests. p values $<0.05$ were considered statistically significant.

The basis for the comparison is a study, which was carried out in the same medical center over a similar time frame (5-years, between 2005 and 2009) [12]; the general and quantitative characteristics, in addition to the instrumentation and identification methods used in the two time periods (2005-2009 and 2013-2017) are summarized in Table 1.

\section{Results}

Between 2013 and 2017, an average of 23,274 2 2,756 blood culture bottles were received per year, out of which $10.5 \%$ presented as culture-positive (including clinically-relevant isolates and contaminants). Overall, 3.3-3.6\% of samples were positive for anaerobes (or $0.4 \%$, if all blood culture bottles are considered), representing 3.5-3.8 anaerobic isolates/1000 blood culture bottles (including both aerobic and anaerobic bottles) per year. The number of hospitalized patients did not change significantly between the two study periods $(\mathrm{p}>0.05)$, however, the number of blood culture bottles processed almost tripled. The relatively drastic decrease in the proportion of positive blood culture bottles $(18.9 \%$ vs. $10.5 \%)$ should be attributed to the significantly higher $(\mathrm{p}=0.008)$ number of bottles received during the second study period (Table 1). Similarly, it may seem that more anaerobic isolates were detected between 2005 and 2009 (4.0-6.3\% of positive blood culture bottles contained anaerobes, $0.7 \%$ in the overall number of submitted blood cultures, representing 5.4-8.7 anaerobic isolates/1000 blood culture bottles [including both aerobic 
Table 1

Comparison of the two respective study periods regarding study population size and methodology.

\begin{tabular}{|c|c|c|}
\hline Study period & 2005-2009 [12] & $2013-2017$ \\
\hline Hospital bed count & $\begin{array}{l}1200 \text { (acute) }+200 \\
\text { (chronic) }\end{array}$ & $\begin{array}{l}1465 \text { (acute) }+355 \\
\text { (chronic) }\end{array}$ \\
\hline Affected population & $\sim 600,000$ patients & $\sim 600,000$ patients \\
\hline $\begin{array}{l}\text { Number of hospitalized } \\
\text { patients (average } \pm \text { SD) }\end{array}$ & $84,043 \pm 570$ & $84,438 \pm 1866$ \\
\hline $\begin{array}{l}\text { Number of blood cultures } \\
\text { bottles processed during } \\
\text { the study period }\end{array}$ & 43,992 & 116,371 \\
\hline $\begin{array}{l}\text { Percentage of positive blood } \\
\text { culture bottles for aerobic } \\
\text { and anaerobic bacteria } \\
\text { overall }\end{array}$ & $18.9 \pm 2.2 \%$ & $10.5 \pm 0.3 \%$ \\
\hline $\begin{array}{l}\text { Percentage of positive blood } \\
\text { culture bottles for } \\
\text { anaerobes (compared to } \\
\text { the overall number of } \\
\text { submitted blood cultures) }\end{array}$ & $0.7 \pm 0.03 \%$ & $0.4 \pm 0.05 \%$ \\
\hline $\begin{array}{l}\text { Number of strict anaerobic } \\
\text { isolates }\end{array}$ & 305 isolates & 423 isolates \\
\hline $\begin{array}{l}\text { Number of strict anaerobic } \\
\text { isolates/1000 } \\
\text { hospitalizations }\end{array}$ & 0.7 & 1.0 \\
\hline $\begin{array}{l}\text { Methods used ifor microbial } \\
\text { identification }\end{array}$ & $\begin{array}{l}\text { Presumptive } \\
\text { identification methods } \\
\text { (Wadsworth Anaerobic } \\
\text { Bacteriology Manual), } \\
\text { Rapid ID 32A } \\
\text { (bioMérieux) }\end{array}$ & $\begin{array}{l}\text { MALDI-TOF MS } \\
\text { (Bruker Daltonics), } \\
\text { extraction with } \\
\text { formic acid before } \\
\text { measurements }\end{array}$ \\
\hline $\begin{array}{l}\text { Blood culture detection } \\
\text { system }\end{array}$ & $\begin{array}{l}\text { BD Bactec (Beckton } \\
\text { Dickinson) }\end{array}$ & $\begin{array}{l}\text { BacT/Alert 3D } \\
\text { (bioMérieux) }\end{array}$ \\
\hline Incubation time & \multicolumn{2}{|c|}{5 days (if endocarditis is suspected: 21 days) } \\
\hline
\end{tabular}

and anaerobic bottles] per year). However, the absolute number of anaerobic isolates $(n=305$ in $2005-2009$ vs. $n=423$ in 2013-2017; $\mathrm{p}=0.018)$ and the number of isolates corresponding to the number of hospitalized patients $(0.7 / 1000$ hospitalizations in $2005-2009$ vs. 1.0 / 1000 hospitalizations in 2013-2017), reveal an increase in the number of anaerobes isolated.

The epidemiological characteristics of affected patients and the indications for blood culture submissions corresponding to blood cultures positive for anaerobes are presented in Table 2 . With regards to the demographic characteristics of the affected patients, the mean age in the present study period was around 70-73 years (range: 18-102 years) and a pronounced female dominance could be observed (male-to-female ratio: 0.60). Compared to 2005-2009, the mean age of patients increased considerably (mean: 60 years, range: 31-84 years) and the gender distribution has also shifted (male-to-female ratio: 1.5). The most prevalent indications for blood culture submissions in the present period were consistent with risk factors described in the literature: cardiovascular diseases (19.9\%), gastrointestinal diseases $(19.3 \%)$, hematological malignancies or solid tumors $(8.0 \%)$, respiratory diseases or pneumonia $(6.4 \%)$, disorders of the urinary system or hemodialysis $(3.7 \%)$ and complications associated with diabetes $(1.1 \%)$. Septicemia was reported in $21.6 \%$ of cases (Table 2 ), in comparison, the number of affected patients on dialysis and reported pneumonia was three times as high as in the previous study period; similarly, sepsis was reported in only $15.9 \%$ of cases between 2005 and 2009. Most blood culture bottles positive for anaerobes (excluding Cutibacterium spp.) originated from the intensive care units $(44.5 \%)$, followed by the department of internal medicine $24.6 \%$ (these departments were predominantly affected in the previous study period as well [12]); the department of neurology $(12.9 \%)$, psychiatry $(9.9 \%)$, surgery $(3.6 \%)$, traumatology and rheumatology (1.3\%, respectively), urology $(0.9 \%)$ and oncology/oncotherapy $(0.8 \%)$ also sent in blood culture bottles positive for anaerobic isolates.

The percentage distribution of isolated anaerobic species in the respective time periods $(2005-2009,2013-2017)$ is presented in Table 3 , while the detailed species distribution of isolates for both time periods is presented in Table 4 . The majority of isolated anaerobes were Cutibacterium (Propionibacterium) spp. $(54.0 \pm 8.5 \%$; $n=247)$, similarly to the previous study period $(56.0 \pm 8.4 \% ; \mathrm{p}>0.05$; Table 3 .). Out of the $n=247$ isolates, none could be considered as clinically significant, according to our criteria. Based on the species distribution of significant anaerobic bacteria in the respective study period, no relevant shift could be observed among isolated species: $(45.8 \%$ vs. $38.9 \%$ for Gram-negatives, $54.2 \%$ vs. $61.1 \%$ for Gram-positives; $\mathrm{p}>0.05$ ), although the ratio of Bacteroides/Parabacteroides isolates increased compared to other Gram-negative anaerobes; members of the Clostridium spp. were the second most common species in both study periods (Tables 3 and 4). Between 2013 and 2017, the isolated anaerobic strains belonged to 38 different anaerobic species, which number is significantly higher $(\mathrm{p}=0.028)$ than in the previous period $(n=26)$. If the levels of identification are compared between the two study periods, it can be observed that $92.2 \%$ of isolates were identified to the species level in the present study period. This ratio in the

Table 2

Epidemiological features of patient population and the indications for blood culture bottle submission (excluding those positive for $C$. acnes) between 2013 and 2017 .

\begin{tabular}{|c|c|c|c|c|c|c|}
\hline Study year & 2013 & 2014 & 2015 & 2016 & 2017 & Overall \\
\hline Number of affected patients & 27 & 28 & 34 & 41 & 57 & 187 \\
\hline Male-to-female ratio & 1.5 & 0.3 & 0.4 & 0.5 & 0.4 & 0.6 \\
\hline Average age $[$ year $\pm \mathrm{SD}]$ & $71.6 \pm 13.5$ & $70.9 \pm 15.6$ & $70.7 \pm 17.8$ & $73.0 \pm 11.6$ & $70.0 \pm 17.1$ & $71.9 \pm 16.7$ \\
\hline Age range [years] & $(42-94)$ & $(25-102)$ & $(19-94)$ & $(47-89)$ & $(18-96)$ & $(18-102)$ \\
\hline Cardiovascular diseases & 4 & 3 & 6 & 13 & 11 & 37 \\
\hline Respiratory diseases, pneumonia & 2 & 0 & 1 & 3 & 6 & 12 \\
\hline Malignancy (solid or hematological) & 4 & 1 & 0 & 4 & 6 & 15 \\
\hline Septicemia & 3 & 10 & 7 & 8 & 12 & 40 \\
\hline Fever & 5 & 1 & 3 & 5 & 5 & 19 \\
\hline Abdominal pain & 8 & 9 & 6 & 0 & 9 & 32 \\
\hline Illness affecting the urinary system, hemodialysis & 1 & 1 & 2 & 1 & 2 & 7 \\
\hline Osteomyelitis & 0 & 2 & 0 & 0 & 0 & 2 \\
\hline Abscess & 0 & 1 & 0 & 1 & 0 & 2 \\
\hline Illness affecting the gastro-intestinal system & 0 & 0 & 4 & 5 & 4 & 13 \\
\hline Type II Diabetes & 0 & 0 & 2 & 0 & 0 & 2 \\
\hline Neurological disease & 0 & 0 & 1 & 1 & 1 & 3 \\
\hline Locomotor disease & 0 & 0 & 2 & 0 & 1 & 3 \\
\hline
\end{tabular}


Table 3

Percentage distribution of anaerobes in the two respective study periods (2005-2009, 2013-2017).

\begin{tabular}{|c|c|c|c|}
\hline Anaerobic isolates & $\begin{array}{l}{ }^{\text {a }} \text { Percentage } \\
\text { of anaerobes } \\
\text { according to } \\
\text { literature } \\
\text { data }^{\text {a }}\end{array}$ & $\begin{array}{l}\text { Results of the } \\
\text { previous } \\
\text { study } \\
(2005-2009) \\
{[12]}\end{array}$ & $\begin{array}{l}\text { Results of } \\
\text { the present } \\
\text { study } \\
(2013-2017)\end{array}$ \\
\hline Cutibacterium spp. ${ }^{b}$ & $\begin{array}{l}30-80 \% \text { (of } \\
\text { isolated } \\
\text { anaerobes) }\end{array}$ & $\begin{array}{l}56.0 \% \\
(n=174)\end{array}$ & $\begin{array}{l}54.0 \% \\
(n=247)\end{array}$ \\
\hline $\begin{array}{l}\text { All other isolates excluding } C l \\
\text { Gram-negative anaerobes }\end{array}$ & acterium spp. & $\begin{array}{l}45.8 \% \\
(n=61)\end{array}$ & $\begin{array}{l}38.9 \% \\
(n=69)\end{array}$ \\
\hline $\begin{array}{l}\text { Bacteroides/Parabacteroides } \\
\text { spp. }\end{array}$ & $26-75 \%$ & $\begin{array}{l}30.6 \% \\
(n=41)\end{array}$ & $\begin{array}{l}34.2 \% \\
(\mathrm{n}=54)\end{array}$ \\
\hline Fusobacterium spp. & $4-15 \%$ & $5.7 \%(\mathrm{n}=8)$ & $1.2 \%(\mathrm{n}=2)$ \\
\hline $\begin{array}{l}\text { Prevotella and } \\
\text { Porphyromonas spp. }\end{array}$ & $0.5-10 \%$ & $5.7 \%(\mathrm{n}=8)$ & $1.2 \%(\mathrm{n}=2)$ \\
\hline Veillonella spp. & $0.5-2 \%$ & $3.7 \%(n=4)$ & $2.3 \%(n=4)$ \\
\hline Gram-positive anaerobes & & $\begin{array}{l}54.2 \% \\
(n=73)\end{array}$ & $\begin{array}{l}61.1 \% \\
(n=107)\end{array}$ \\
\hline Clostridium spp. & $8-46 \%$ & $\begin{array}{l}30.6 \% \\
(n=41)\end{array}$ & $\begin{array}{l}33.3 \% \\
(\mathrm{n}=59)\end{array}$ \\
\hline $\begin{array}{l}\text { Gram-positive anaerobic } \\
\text { cocci (GPAC) }\end{array}$ & $8-20 \%$ & $\begin{array}{l}17.9 \% \\
(\mathrm{n}=24)\end{array}$ & $\begin{array}{l}12.0 \% \\
(\mathrm{n}=21)\end{array}$ \\
\hline $\begin{array}{l}\text { Gram-positive non-spore } \\
\text { forming rods (excluding: } \\
\text { Cutibacterium spp.) }\end{array}$ & $0.5-14 \%$ & $5.7 \%(\mathrm{n}=8)$ & $\begin{array}{l}15.8 \% \\
(n=27)\end{array}$ \\
\hline
\end{tabular}

a See References.

b Corresponding to all Cutibacterium spp. (btoh contaminants and clinically-relevant isolates).

$2005-2009$ period was only $77.6 \%(p=0.031)$. Polymicrobial anaerobic bacteremia is considered very rare, which is further highlighted by our results: over the 5-year period (2013-2017), two anaerobes were isolated simultaneously in five cases (Bacteroides spp. or Clostridium spp., together with a GPAC), while in 14 cases, one anaerobic strain (Bacteroides spp. or Clostridium spp.) was co-isolated with a facultative anaerobic bacteria (Escherichia coli, Klebsiella pneumoniae, Staphylococcus aureus, S. epidermidis).

The time-to-positivity (TTP) in blood cultures positive for anaerobes was $31.4 \pm 23.4 \mathrm{~h}$ for pathogens other than Cutibacterium spp., but much longer $(112.9 \pm 37.2 \mathrm{~h})$ for Cutibacterium spp. isolates, $(\mathrm{p}<0.0001)$.

\section{Discussion}

Despite their relatively low prevalence, anaerobic bacteria are important etiological factors in bloodstream infections and other invasive infections. The knowledge of relevant risk factors is important to maintain a degree of suspicion in clinicians and in selecting appropriate empirical antibiotic therapy. The local prevalence and species distribution in this study is in line with the literature data $(\sim 1.0 / 1000$ hospitalized patients, $0.5-13 \%$ of positive blood cultures, most often Bacteroides/Parabacteroides spp. and Clostridium spp.), however, due to the absence of other local data in Hungary, we were not able to draw relevant national or institutional comparisons. Compared to the previous five-year study period (2005-2009), the absolute number of anaerobic isolates increased, both in respect to the number of individual isolates and in standardized values for hospitalized patients, although this may seem proportionally lower, due to a significant increase in the number of blood culture bottles submitted [12]. The disproportionally high number of positive blood cultures originating from the ICUs may be attributable to the logistical setup of the Clinical Center (a larger, logistically more coherent single adult ICU has been created in one place, instead of them being scattered). In the previous study, the 30 -day crude mortality rate was determined $(22.3 \%)$ [12], however, in the present study, we did not have the opportunity to observe the mortality rate, as the medical charts of the individual patients affected were not available during the survey.

Of particular interest is the appearance of various rare anaerobic species between 2013 and 2017, such as Actinotignum schaali, Collinsella aerofaciens, Flavonifractor plautii, Solobacterium moorei, and Tissierella praeacuta. Some of these microorganisms have previously been designated into different genera (i.e. A. schaali was formerly known as Actinobaculum schaalii, C. aerofaciens and $F$. plautii were both designated in the Eubacterium genus, T. praeacuta was previously Bacteroides praeacutus), while S. moorei (an identified contributor to halitosis) has only been known since the 2000s. Their increasing detection rate is probably due to the growing number of laboratories nowadays which have the capabilities (polymerase chain reaction, MALDI-TOF MS, $16 \mathrm{~S}$ rRNA sequencing) that allow for their accurate species level identification $[17,18]$. Additionally, an increasing spectrum of organisms detected is also due to advances in taxonomy, as many are being reclassified into different genera. In the present study, all Cutibacterium spp. isolates were considered contaminants (based on our criteria described in the Results section), however, their clinical role is not to be dismissed as they are increasingly being reported in the literature as potential pathogens [19]. C. acnes is a member of the normal skin flora, and its role in the development of acne vulgaris is well-known [20]; it is a very common contaminant in blood cultures due to their anatomical location. Nevertheless, more and more studies address its role in endocarditis, shunt infections, orthopedic infections (e.g., knee or hip replacement) and ocular infections and improved diagnostic methods may also aid in their potential identification as pathogens [19].

A change in the blood culture detection systems (BD Bactec vs. BacT/Alert 3D) occurred between the two respective study periods; there is a large body of evidence demonstrating that automated systems using different detection methods for $\mathrm{CO}_{2}$ (colorimetric/fluorescence measurement) have different efficacy in detecting anaerobic bacteria [21]. Fiori et al. demonstrated that BacT/Alert systems showed a better recovery of Gram-positive microorganisms (both aerobes and strict anaerobes), while higher efficacy in the recovery of Gram-negative bacteria was observed for the BACTEC system [22]; nonetheless, significant shifts towards either Gram-positive or Gram-negative bacteria were not observed in our data, if the isolation frequency of Gram-positive and Gram-negative microorganisms are taken into consideration. In contrast, the study of Jeverica et al. showed that BACTEC systems with lytic bottles were more effective and consistent in the detection of Cutibacterium spp. than BacT/ Alert systems [23]. In addition, some manufacturers' bottles may contain components (such as sodium polyanethylene sulfonate or SPS) that have been shown to inhibit the growth of certain anaerobic bacteria [24]. In our settings, blood culture bottles were changed in parallel with the detection systems; however the bottles used in the Institute reportedly do not contain such substances (based on manufacturer's specifications), therefore it is unlikely to have affected the isolation rate and composition of strict anaerobes for this reason. Nonetheless, it should be pointed out that the different size, shape and handling requirements of the different blood culture bottles for acquiring samples aseptically, may have an effect on the ratio of contaminants (i.e. Cutibacterium spp.); however, relevant differences were also not demonstrated ( $56.0 \%$ vs. $54.0 \%$ of overall isolates) in this regard, between the two periods. Parallel to the change the blood culture equipment, practical trainings were done time and again for appropriate sampling methods for all of the clinical staff, especially the nurses, 
Table 4

Detailed characterization of anaerobic isolates from blood culture bottles (2005-2009 and 2013-2017).

\begin{tabular}{|c|c|c|c|c|c|c|c|}
\hline Study year & $2005-2009$ & 2013 & 2014 & 2015 & 2016 & 2017 & 2013-2017 \\
\hline Number of anaerobic isolates & 305 & 72 & 76 & 85 & 86 & 104 & 423 \\
\hline Microorganisms identified to the species level & 26 & 14 & 12 & 15 & 13 & 26 & 38 \\
\hline \multicolumn{8}{|l|}{ Gram-positive, spore-forming anaerobic rods } \\
\hline Clostridium spp. (genus level) & 13 & 0 & 1 & 0 & 0 & 0 & 1 \\
\hline C. butyricum & 4 & 0 & 0 & 0 & 0 & 0 & 0 \\
\hline C. clostridioforme & 0 & 1 & 0 & 0 & 0 & 0 & 1 \\
\hline C. perfringens & 21 & 7 & 5 & 8 & 8 & 8 & 36 \\
\hline C. septicum & 3 & 1 & 0 & 1 & 0 & 1 & 3 \\
\hline C. sordelii & 0 & 1 & 0 & 1 & 0 & 1 & 3 \\
\hline C. tertium & 0 & 1 & 0 & 0 & 0 & 0 & 1 \\
\hline C. paraputrificum & 0 & 0 & 1 & 0 & 1 & 1 & 3 \\
\hline C. ramosum & 0 & 0 & 2 & 0 & 1 & 1 & 4 \\
\hline C. innocuum & 2 & 0 & 0 & 2 & 0 & 1 & 3 \\
\hline C. sporogenes & 0 & 0 & 0 & 0 & 0 & 1 & 1 \\
\hline C. symbiosum & 0 & 0 & 0 & 0 & 0 & 2 & 2 \\
\hline C. hathewayi & 0 & 0 & 0 & 0 & 0 & 1 & 1 \\
\hline \multicolumn{8}{|l|}{ Gram-positive, non-spore-forming anaerobic rods } \\
\hline Actinotignum schaali & 0 & 0 & 0 & 0 & 0 & 1 & 1 \\
\hline Actinomyces spp. (genus level) & 1 & 0 & 0 & 0 & 0 & 0 & 0 \\
\hline A. odontolyticus & 0 & 0 & 1 & 1 & 1 & 1 & 3 \\
\hline A. neuii & 0 & 0 & 0 & 1 & 0 & 0 & 1 \\
\hline Bifidobacterium pseudocatenulatum & 0 & 0 & 0 & 1 & 0 & 0 & 1 \\
\hline B. dentium & 0 & 0 & 0 & 1 & 0 & 0 & 1 \\
\hline Cutibacterium (Propionibacterium) spp. (genus level) & 1 & 1 & 8 & 4 & 1 & 5 & 19 \\
\hline C. acnes & 171 & 42 & 40 & 52 & 50 & 41 & 225 \\
\hline C. avidum & 0 & 1 & 0 & 1 & 0 & 0 & 2 \\
\hline C. granulosum & 2 & 1 & 0 & 0 & 0 & 0 & 1 \\
\hline Collinsella aerofaciens & 0 & 0 & 0 & 0 & 0 & 1 & 1 \\
\hline Eggerthella lenta & 3 & 1 & 1 & 0 & 4 & 1 & 7 \\
\hline Eubacterium limosum & 0 & 0 & 0 & 0 & 0 & 2 & 2 \\
\hline Flavonifractor plautii & 0 & 0 & 0 & 0 & 1 & 0 & 1 \\
\hline Lactobacillus spp. (genus level) & 12 & 2 & 3 & 2 & 1 & 1 & 9 \\
\hline Solobacterium moorei & 0 & 0 & 0 & 0 & 1 & 1 & 2 \\
\hline \multicolumn{8}{|l|}{ Gram-positive anaerobic cocci (GPAC) } \\
\hline Anaerococcus spp. & 0 & 0 & 0 & 0 & 0 & 1 & 1 \\
\hline Finegoldia magna & 2 & 0 & 0 & 0 & 1 & 1 & 2 \\
\hline Parvimonas micra & 10 & 0 & 1 & 1 & 3 & 6 & 11 \\
\hline Peptinophilus asaccharolyticus & 9 & 0 & 0 & 0 & 0 & 0 & 0 \\
\hline P. harei & 0 & 0 & 1 & 2 & 0 & 3 & 6 \\
\hline Peptostreptococcus spp. (genus level) & 0 & 0 & 1 & 0 & 0 & 0 & 1 \\
\hline P. anaerobius & 3 & 0 & 0 & 0 & 0 & 0 & 0 \\
\hline \multicolumn{8}{|l|}{ Gram-negative anaerobic rods } \\
\hline Bacteroides spp. (genus level) & 8 & 0 & 1 & 0 & 0 & 0 & 1 \\
\hline B. fragilis & 14 & 9 & 8 & 5 & 11 & 14 & 47 \\
\hline B. caccae & 2 & 0 & 0 & 0 & 0 & 0 & 0 \\
\hline B. vulgatus & 0 & 1 & 1 & 0 & 0 & 0 & 1 \\
\hline B. thetaiotaomicron & 6 & 0 & 1 & 1 & 0 & 1 & 3 \\
\hline B. merdae & 1 & 0 & 0 & 0 & 0 & 0 & 0 \\
\hline B. ovatus & 0 & 0 & 0 & 0 & 0 & 1 & 1 \\
\hline B. pyogenes & 1 & 0 & 0 & 0 & 0 & 1 & 1 \\
\hline Fusobacterium nucleatum & 8 & 0 & 0 & 0 & 0 & 0 & 0 \\
\hline F. necrophorum & 2 & 1 & 0 & 0 & 0 & 1 & 2 \\
\hline Prevotella buccae & 3 & 0 & 0 & 0 & 0 & 0 & 0 \\
\hline P. denticola & 2 & 1 & 0 & 0 & 0 & 0 & 1 \\
\hline P. melaninogenica & 1 & 0 & 0 & 1 & 0 & 0 & 1 \\
\hline P. oralis & 1 & 0 & 0 & 0 & 0 & 0 & 0 \\
\hline Tissierella praeacuta & 0 & 0 & 0 & 0 & 0 & 1 & 1 \\
\hline \multicolumn{8}{|l|}{ Gram-negative anaerobic cocci } \\
\hline Veillonella atypica & 0 & 1 & 0 & 0 & 1 & 1 & 3 \\
\hline$V$. dispar & 0 & 0 & 0 & 0 & 1 & 0 & 1 \\
\hline V. parvula & 3 & 0 & 0 & 0 & 0 & 0 & 0 \\
\hline
\end{tabular}

ICU practitioners, residents and nursekeepers. This included the strict adherence to Instuttional and international guidelines and sample ordering practices for blood culture bottles (i.e. to order two sets of one aerobic and one anaerobic blood culture bottle per set), which was not necessary the case in the previous study period [12].

We have also collected data on blood culture TTP, associated with the isolation of anaerobes: in many cases, TTP may provide clinically relevant information to the physicians, regarding the pathogen/contaminant status of the isolated species. Correlation between clinical outcome of patients and TTP values has been reported previously [25]. Based on our present observations and in accordance with the literature, a "threshold" of approximately $60 \mathrm{~h}$ of TTP may be established, where the isolated anaerobic species is no longer expected to be clinically significant, based on our criteria (taking into account the 
underlying illnesses of the patient), aiding physicians in the choice of empiric antibiotic therapy. However, it is also important to note that some Prevotella and Porphyromonas species may have TTP values up to $80-100 \mathrm{~h}$ (due to their slow generation times, especially if they are represented in the sample with low colony forming unit count [26]), which overlaps with the TTP values of Cutibacterium spp. In our settings, this was not observed as a confounding factor (the TTP values of Prevotella and Porphyromonas species were below $55 \mathrm{~h}$ in every case).

A number of different studies reported on the recovery of anaerobes in patients with bacteremia during the last several decades [1,5-7]; however, at the same time, conflicting data has accumulated regarding the incidence of anaerobic bacteremia. Earlier studies showed that anaerobes account for around $20 \%$ of all cases bacteremia [26], however, more recent data suggest that obligate anaerobic bacteria account for about $5 \%$ of bacteremia (range: $0.5 \%-13 \%$ of bacteremias; approximately $0.1 \%$ of hospital admissions) $[1,4,6,7,10,12]$. There may be multiple reasons explaining the different observations in other studies regarding the epidemiology of anaerobic bacteremia $[27,28]$ : the re-emergence of anaerobic bacteremia may depend on antibiotic policies of the hospital, the different geographical regions and differences in the study population, including the patient age, immune status, prevalence and severity of underlying diseases, social status and other factors (such as malnutrition or on the contrary, obesity). Older patients seem to be at increased risk for developing anaerobic bacteremia, while children, especially between 2 and 5 years of age have the lowest risk [29].

In the last decade, the decrease in the incidence of anaerobic bacteremia was reported in some publications [28,30-32]. In contrast to these reports, other publications reported a considerable increase in the incidence of anaerobic bacteremia. In the pivotal publication by Cockerill et al., corresponding to the period between 1984 and 1992, a considerable increase in the incidence of anaerobic bacteremia was noted at the Mayo Clinic [33] and later, another retrospective study report from the same institution also observed an increase in incidence during the subsequent 12-year period (from 1993 through 2004) [28]. In a 12-year study at an Australian general hospital, Riley and Arvavena [34] found a $200 \%$ increase in the incidence of anaerobic bacteremia, with Fusobacterium spp. and Gram-positive anaerobic cocci (GPAC) being more frequently identified. Other investigators have also reported increases in the incidence of anaerobic bacteremia, particularly during the late 1990s and early 2000s [35-37]. Reasons for this increase were unclear: some authors hypothesized that the routine use of inappropriate antibiotic prophylaxis and/or bowel preparations prior to abdominal surgery may explain this phenomenon. Because the decrease/increase in the incidence of anaerobic bacteremia varied from study to study, selective rather than routine use of an anaerobic bottle for culturing blood samples has been proposed in the medical literature. According to the suggestions of Badri et al. [14], is not necessary to detect anaerobic bacteremia in patients by microbiological methods, as the patients could be identified clinically; and if it is likely that they will have anaerobic bacteremia with a high degree of predictability, they should be treated empirically without the need for microbiological confirmation. The utility of anaerobic blood cultures remains a controversial topic, while reports suggesting that anaerobic sepsis, especially in patients with relevant risk factors and severe underlying diseases is becoming more prevalent. According to our point of view and our current clinical observations, routine anaerobic blood cultures should not be abandoned. Additionally, the increasing resistance rates in anaerobes and the presence of multidrug resistant isolates (most commonly in $\mathrm{Bac}$ -
teroides/Parabacteroides spp.) are also important reasons for performing anaerobic bacteriology in cases of possible or suspected anaerobic bacteremia.

Only a small number of comparative studies can be found in the literature on the incidence of anaerobic bacteremia in the same study site over different periods of time. According to Dorsher et al., the incidence of anaerobic bacteremia from 1974 to 1988 at the Mayo Clinic (Rochester, MN), decreased by $45.0 \%$ [31], in addition, the positive rate of blood cultures for anaerobes decreased significantly, even though the total number of submitted blood cultures increased. The number of anaerobic bacteremias per 100,000 patient-days also declined over this 15-year period. Strains of the Bacteroides/Parabacteroides genus ranked third in frequency if compared to other organisms causing aerobic and anaerobic bacteremia in 1974, while they were only the seventh most common bacteria in 1988, causing slightly less than half of the anaerobic bacteremias. The mechanisms responsible for these changes are unclear, but might relate to earlier recognition and treatment of localized anaerobic infection, widespread preoperative use of agents prior to bowel surgery, and use of broad-spectrum antimicrobial regimens that included agents with activity against anaerobes. A later study from the same hospital indicated the re-emergence of anaerobic bacteremia in the 12-year period from 1993 through 2004 [14]. Medical records for patients with anaerobic bloodstream infections were analysed throughout the study period to identify differences between these two patient populations with different rates of bacteremia. The number of anaerobic blood cultures per 1000 cultures performed increased by $30 \%$. The mean incidence of anaerobic bacteremias increased from $n=53$ cases per year during 1993-1996, to $n=75$ cases per year during 1997-2000, followed by $n=91$ cases per year during 2001-2004; the total number of cases of anaerobic bacteremia per 100,000 patient-days increased by $74 \%$. They reported the following species-distribution from the positive anaerobic blood cultures in the different time periods: Bacteroides/ Parabacteroides spp. were most commonly isolated (26.0-43.0\%), other anaerobic Gram-negative bacteria in $8-25 \%$, Prevotella and Porphyromonas spp. in 2\%-10\%, GPAC in 20-35\%, Clostridium spp.in $16 \%-46 \%$ and non-spore forming Gram-positive bacteria in $4 \%-18 \%$, respectively (1993-2004). Their data showed a striking revelation: $38 \%$ of patients with anaerobic bacteremia in 2004 had sources other than the genito-urinary and/or gastrointestinal tracts and by way of warning that in $34.3 \%$ of patients, anaerobes would have not been suspected as the cause of bacteremia on the basis of "typical" clinical predictors. They concluded that sources of anaerobic bacteremia are now more varied than previously described, especially among older and immunosuppressed patients and those patients who suffered from complex underlying disease.

\section{Conclusions}

Anaerobic blood cultures may be helpful when anaerobic bacteremia is clinically suspected, i.e. in patients with advanced age and/ or in severely immunocompromised state, with serious underlying diseases, in which case the correct source of bacteremia is not identified by clinical evaluation. The prevalence of anaerobic bacteremia in relation to patient demographics should be determined on an institutional basis to guide blood-culture practices. This approach is important to provide timely and optimal treatment for patients. The use of modern diagnostic modalities (MALDI-TOF MS, PCR) in routine anaerobic diagnostics may aid in getting a better view into the frequency of anaerobic bacteria isolated from blood. 


\section{Author contributions}

M.G. and E.U. conceived and designed the study. E.U. was the senior microbiologist, head of the Hungarian National Anaerobe Reference Laboratory in the study period, performing bacterial isolation and identification. M.Á., A.L. and G.T. performed bacterial isolation and identification. M.G. performed data collection and analysis. E.U. performed the literature survey for writing the article. M.G., M.Á., A.L., G.T. and E.U. wrote and revised the full paper. All authors have read and agreed to the published version of the manuscript.

\section{Funding}

This research received no external funding.

\section{Declaration of competing interest}

The author declares no conflicts of interest, monetary or otherwise.

\section{Acknowledgments}

The authors would like to acknowledge the staff of the Institute of Clinical Microbiology, University of Szeged. M.G. was supported by the National Youth Excellence Scholarship [Grant Number NTP-NTFÖ-18-C-0225] and the ESCMID Mentorship and Observership Programme. Part of this study was presented at the 2018 General Meeting of the Hungarian Society for Microbiology (Eger, Hungary).

\section{References}

[1] I. Brook, The role of anaerobic bacteria in bacteremia, Anaerobe 16 (2010) 183-189.

[2] M. Gajdács, G. Spengler, E. Urbán, Identification and antimicrobial susceptibility testing of anaerobic bacteria: rubik's cube of clinical microbiology?, Antibiotics 6 (2017) e25.

[3] J.A. Washington, Comparison of two commercially available media for detection of bacteremia, Appl. Microbiol. 22 (1971) 604-607.

[4] A. Arzese, R. Trevisan, M.G. Menozzi, Anaerobe-induced bacteremia in Italy: a nationwide survey. The Italian anaerobe study group, Clin. Infect. Dis. 20 (Supp 2) (1995) S230-S232.

[5] F. Vazquez, F.J. Mendez, F. Perez, M.C. Mendoza, Anaerobic bacteremia in a general hospital: retrospective five-year analysis, Rev. Infect. Dis. 9 (1987) 1038-1043.

[6] V.A. Peraino, S.A. Cross, E.J. Goldstein, Incidence and clinical significance of anaerobic bacteremia in a community hospital, Clin. Infect. Dis. 16 (1993) S288-S291.

[7] J.R. Wilson, A.P. Limaye, Risk factors for mortality in patients with anaerobic bacteremia, Eur. J. Clin. Microbiol. Infect. Dis. 23 (2004) 310-316.

[8] E. Nagy, L. Boyanova, U.S. Justesen, ESCMID Study Group of Anaerobic Infections. How to isolate, identify and determine antimicrobial susceptibility of anaerobic bacteria in routine laboratories, Clin. Microbiol. Infect. 24 (2018) $1139-1148$.

[9] J. Sóki, M. Hedberg, S. Patrick, B. Bálint, R. Herczeg, I. Nagy, D.W. Hecht, E. Nagy, E. Urbán, Emergence and evolution of an international cluster of MDR Bacteroides fragilis isolates, J. Antimicrob. Chemother. 71 (2016) 2441-2448

[10] J.H. Salonen, E. Eerola, O. Meurman, Clinical significance and outcome of anaerobic bacteremia, Clin. Infect. Dis. 26 (1998) 1413-1417.

[11] C.W. Cheng, H.S. Lin, J.J. Ye, C.C. Yang, P.C. Chiang, T.S. Wu, M.H. Lee, Clinical significance of and outcomes for Bacteroides fragilis bacteremia, J. Microbiol. Immunol. Infect. 42 (2009) 243-250.

[12] E. Urbán, Five-year retrospective epidemiological survey of anaerobic bacteremia in a university hospital and rewiew of the literature, Eur J Microbiol Immunol 2 (2012) 140-147.

[13] Hospital bed count and patient turnover report 2017. National health insurance fund of Hungary, Available from http://www.neak.gov.hu/felso_menu/szakmai oldalak/publikus_forgalmi_adatok/gyogyito_megelozo_forgalmi_adat/ fekvobeteg_szakellatas/korhazi_agyszam.html, Accessed on 4th of January 2020
[14] M. Badri, B. Nilson, S. Ragnarsson, E. Senneby, M. Rasmussen, Clinical and microbiological features of bacteraemia with Gram-positive anaerobic cocci: a population-based retrospective study, Clin. Microbiol. Infect. 25 (2019) 760, e1-760.e6.

[15] T.J. Kirn, M.P. Weinstein, Update on blood cultures: how to obtain, process, report, and interpret, Clin. Microbiol. Infect. 19 (2013) 513-520.

[16] M. Gajdács, E. Urbán, The relevance of anaerobic bacteria in brain abscesses: a ten-year retrospective analysis (2008-2017), Inf. Disp. 51 (2019) 779-781.

[17] S. Shannon, D. Kronemann, R. Patel, A.N. Schuetz, Routine use of MALDI-TOF MS for anaerobic bacterial identification in clinical microbiology, Anaerobe 54 (2018) 191-196.

[18] O. Opota, A. Croxatto, G. Prod'hom, G. Greub, Blood culture-based diagnosis of bacteraemia: state of the art, Clin. Microbiol. Infect. 21 (2015) 313-322.

[19] H.J. Park, S. Na, S.Y. Park, S.M. Moon, O.H. Cho, K.H. Park, Y.P. Chong, S.H. Kim, S.O. Lee, Y.S. Kim, J.H. Woo, M.N. Kim, S.H. Choi, Clinical significance of propionibacterium acnes recovered from blood cultures: analysis of 524 episodes, J. Clin. Microbiol. 49 (2011) 1598-1601.

[20] I. Nagy, A. Pivarcsi, A. Koreck, M. Széll, E. Urbán, L. Kemény, Distinct strains of Propionibacterium acnes induce selective human $\beta$-defensin- 2 and interleukin-8 expression in human keratinocytes through toll-like receptors, J. Invest. Dermatol. 124 (2005) 931-938.

[21] M. Mueller-Premru, S. Jeverica, L. Papst, E. Nagy, Performance of two blood culture systems to detect anaerobic bacteria. Is there any difference?, Anaerobe 45 (2017) 59-64.

[22] B. Fiori, T. D'Inzeo, V. Di Florio, F. DeMaio, G. De Angelis, A. Giaquinto, L. Campana, E. Tanzarella, M. Tumbarello, M. Antonelli, M. Sanguinetti, T. Spanu, Performance of two resin containing blood culture media in detection of bloodstream infections and in direct matrix-assisted laser desorption ionization-time of flight mass spectrometry (MALDI-TOF MS) broth assays for isolate identification: clinical comparison of the BacT/Alert Plus and Bactec Plus systems, J. Clin. Microbiol. 52 (2014) 3558-3567.

[23] S. Jeverica, F.E. Sayed, P. Camernik, B. Kocjacic, B. Sluga, M. Rottman, L. Papst, Growth detection of Cutibacterium acnes from orthopaedic implantassociated infections in anaerobic bottles from BACTEC and BacT/ALERT blood culture systems and comparison with conventional culture media, Anaerobe 61 (2020), 102133.

[24] M.H. Graves, J.A. Morello, F.E. Kocka, Sodium polyanethol sulfonate sensitivity of anaerobic cocci, Appl. Microbiol. 27 (1974) 1131-1133.

[25] B. Lamy, Blood culture time-to-positivity: making use of the hidden information, Clin. Microbiol. Infect. 25 (2019) 268-271.

[26] S.M. Finegold, Anaerobic Bacteria in Human Disease, Academic Press, New York, 1977.

[27] E. Ortiz, M.A. Sande, Routine use of anaerobic blood cultures: are they still indicated, Am. J. Med. 108 (2000) 445-447.

[28] B. Lassmann, D.R. Gustafson, C.M. Wood, J.E. Rosenblatt, Reemergence of anaerobic bacteremia, Clin. Infect. Dis. 44 (2007) 895-900.

[29] I. Brook, Clinical review: bacteremia caused by anaerobic bacteria in children, Crit. Care 6 (2002) 205-211.

[30] D.P. Lombardi, N.C. Engleberg, Anaerobic bacteremia: incidence, patient characteristics, and clinical significance, Am. J. Med. 92 (1992) 53-60.

[31] C.W. Dorsher, J.E. Rosenblatt, W.R. Wilson, D.M. Ilstrup, Anaerobic bacteremia: decreasing rate over a 15-year period, Rev. Infect. Dis. 13 (1991) 633-636.

[32] M.P. Weinstein, M.L. Towns, S.M. Quartey, S. Mirrett, L.G. Reimer, G. Parmigiani, L.B. Reller, The clinical significance of positive blood cultures in the 1990s: a prospective comprehensive evaluation of the microbiology, epidemiology, and outcome of bacteremia and fungemia in adults, Clin. Infect. Dis 24 (1997) 584-602.

[33] F.R. Cockerill, J.G. Hughes, E.A. Vetter, R.A. Mueller, A.L. Weaver, D.M. Ilstrup, J.E. Rosenblatt, W.R. Wilson, Analysis of 281,797 consecutive blood cultures performed over an eight-year period: trends in microorganisms isolated and the value of anaerobic culture of blood, Clin. Infect. Dis. 24 (1997) 403-418.

[34] T.V. Riley, M.A. Aravena, Anaerobic bacteremia in an Australian teaching hospital, Eur. J. Clin. Microbiol. Infect. Dis. 14 (1995) 73-75.

[35] S. Spanik, J. Trupl, A. Kunova, P. Pichna, L. Helpiasnska, I. Ilavska, E. Kukuckova, J. Lacka, S. Grausova, K. Stopokova, L. Drogna, V. Krcméry Jr., Bloodstream infections due to anaerobic bacteria in cancer patients: epidemiology, etiology, risk factors, clinical presentation and outcome of anaerobic bacteremia, Neoplasma 43 (1996) 235-238.

[36] M.H. Nguyen, V.L. Yu, A.J. Morris, L. McDermott, M.W. Wagener, L. Harrell, D.R. Snydman, Antimicrobial resistance and clinical outcome of Bacteroides bacteremia: findings of a multicenter prospective observational trial, Clin. Infect. Dis. 30 (2000) $870-876$.

[37] B. Lorber, Bacteroides, Prevotella, Porphyromonas, and Fusobacterium species (and other medically important anaerobic Gram-negative bacilli), in: G.L. Mandell, J.E. Bennett, R. Dolin (Eds.), Principles and Practice of Infectious Diseases, fifth ed.s, Churchill Livingstone, Philadelphia, PA, 2000, pp. 2561-2570. 